\title{
Telemedicine and Computer-Based Technologies during Coronavirus Disease 2019 Infection; A Chance to Educate and Diagnose
}

\author{
Reza Jafarzadeh-Esfehani, MD ${ }^{1,2}$; Mohsen Mirzaei Fard, MSc ${ }^{3}$; Farzaneh Habibi Hatam-Ghale, MSc ; Alireza Rezaei Kalat, MSc ${ }^{5}$; Amir Fathi, \\ MSc'; Mohammad Shariati, MD²,6; Ariane Sadr-Nabavi, PhD ${ }^{1,2}$; Rahele Miri, MD²; Hamid Reza Bidkhori, MD, PhD ${ }^{2 *}$; Mohammad Hassan \\ Aelami, $\mathrm{MD}^{7^{*}}$ \\ 'Department of Medical Genetics and Molecular Medicine, Faculty of Medicine, Mashhad University of Medical Sciences, Mashhad, Iran \\ 2 Blood Borne Infections Research Center, Academic Center for Education, Culture, and Research (ACECR)-Khorasan Razavi, Mashhad, Iran \\ ${ }^{3}$ Department of Engineering, Faculty of Electrical \& Electronics Engineering, Ferdowsi University, Mashhad, Iran \\ ${ }^{4}$ Department of Engineering, Faculty of Computer Engineering, Khayyam University, Mashhad, Iran \\ ${ }^{5}$ Department of Chemical and Petroleum Engineering, Sharif University of Technology, Tehran, Iran \\ ${ }^{6}$ Department of Neurology, Faculty of Medicine, Mashhad University of Medical Sciences, Mashhad, Iran \\ ${ }^{7}$ Department of Pediatrics and Hand Hygiene and Infection Control Research Center, Imam Reza Hospital, Mashhad University of Medical \\ Sciences, Mashhad, Iran
}

\begin{abstract}
Coronavirus disease 2019 (COVID-19) is now of global concern due to its rapid dissemination across the globe. The rapid spread of this viral infection, along with many of its unknown aspects, has posed new challenges to the health care systems. The main challenging effects of COVID-19 are rapid dissemination through close contact and varying clinical severity among different individuals. Furthermore, the medical staff in endemic areas are becoming exhausted and deal with a considerable level of job burnout, which can negatively affect their medical decision making. Also, due to the variable pulmonary manifestations of COVID-19, some physicians may misdiagnose patients. To overcome these issues, we proposed a web-based software to aid physicians in detecting possible COVID-19 cases through online consultation with different specialists and educate the not-well experienced physicians. Our results demonstrated that this software could improve the diagnostic rate for not-well experienced physicians.

Keywords: Coronavirus, Pandemics, Telemedicine

Cite this article as: Jafarzadeh-Esfehani R, Mirzaei Fard M, Habibi Hatam-Ghale F, Rezaei Kalat A, Fathi A, Shariati M, et al. Telemedicine and computer-based technologies during coronavirus disease 2019 infection; a chance to educate and diagnose. Arch Iran Med. 2020;23(8):561-563. doi: 10.34172/aim.2020.60.
\end{abstract}

Received: April 30, 2020, Accepted: June 2, 2020, ePublished: August 1, 2020

\section{Introduction}

Since the recent coronavirus 2019 (COVID-19) outbreak, many different health issues have been raised. It is now well demonstrated that many of the suspected and confirmed cases in Wuhan city, China had close contact with infected patients (even when they were asymptomatic or had mild symptoms) and medical caregivers, as well as family members of the infected persons, are now considered as a high-risk group. ${ }^{1}$ During the early phase of the outbreak, many hospitals had to limit other services to meet the hospitalization needs of infected patients, and medical staff became exhausted, covering the considerable number of patients referred to hospitals. ${ }^{1}$ This situation highlights the three most important issues regarding the COVID-19 outbreak. These issues include (i) the importance of effective disease control and case tracing, (ii) reducing the pressure on the medical staff and health care workers to improve their medical judgment and performance abilities, and (iii) educating the physicians who have not previously encountered COVID-19 cases and are not familiar with their clinical and radiological presentations. ${ }^{2}$ In such pandemic situations, telemedicine may be a possible solution for health-care services dealing with the mentioned issues. Although it has been reported that using telemedicine during the COVID-19 outbreak may cause challenges in providing effective health care delivery, ${ }^{3}$ we believe that telemedicine may help with providing timely and effective consultation as well as practical education for both well experienced and other less experienced physicians in diagnosing COVID-19 patients. In the present report, we will discuss our experience of developing software for diagnosing COVID-19 patients and educating the physicians for better diagnosis of this novel disease.

*Corresponding Author: Hamid Reza Bidkhori, MD, PhD; Blood Borne Infections Research Center, Academic Centre for Education, Culture, and Research (ACECR)-Khorasan Razavi, University Campus, Azadi sq., Mashhad, Iran. Email: bidkhori@acecr.ac.ir 


\section{Material and Methods}

To minimize the misdiagnosis of COVID-19 patients, our multidisciplinary team recently developed the idea of using telemedicine to provide better diagnosis and increase the experience of medical staff who are dealing with the novel coronavirus disease (Figure 1). In some countries with sufficient access to specific laboratory testing for COVID-19, patients may first undergo laboratory testing rather than imaging study. However, the detection of susceptible cases in the outbreak settings would be problematic. In such situations, the medical staff are dealing with a considerable load of patients who are suffering from various symptoms, including fever and respiratory symptoms. Effective and rapid triage of such patients requires a multidisciplinary team to detect the possible COVID-19 patients and discharge or admit other patients in specific wards. In our opinion, timely and effective diagnosis of COVID-19 in such overwhelming settings requires a multidisciplinary team consisting of specialists in the fields of pulmonology, radiology, and infectious diseases. Our proposed web-based software provides a virtual workspace for two kinds of users, including specialists (radiologists, pulmonologists and infectious disease specialists) and general users (general physicians or specialists from other fields of medicine). The general user can upload the medical information of a suspicious case and provide chest X-rays or chest computed tomography scans. The software will automatically make a medical record for each patient and simultaneously send the record to specialists. Specialists will then evaluate each record anonymously and send their possible diagnosis to the general user. Moreover, they may add medical notes based on their answers, either indicating differential diagnoses or providing diagnostic educational notes for the general users.

\section{Results}

To test our software, 20 physicians (general users) who first visited suspicious COVID-19 patients and were not well experienced in diagnosing COVID-19 were chosen and asked to provide their diagnosis according to the clinical and imaging findings. Then, they were asked to upload their cases' clinical and imaging data into the software. The cases were sent anonymously to specialists in fields of pulmonology, infectious diseases, and radiology at the same time, and all of them provided their clinical diagnosis as well as a clinical note. Then, the general users were asked to provide their final diagnosis for their patients according to the consultations. The correct diagnosis for each patient was then provided to both specialists and general users. The public users could only diagnose $50 \%$ of the submitted cases, and when they considered the simultaneous consultation from the six specialists, their diagnosis rate rose to $90 \%$. Among the specialists, from a total of 120 responses, $84.16 \%$ was the correct diagnosis.

In real-life settings and not in the study settings, based on the clinical condition of the patient, the general user decides to finalize the case and provide a definite diagnosis whenever the diagnosis is confirmed. At this time, every specialist will be able to see the final diagnosis of the patient and also the other specialists' comments. So, both the general user and the specialists will be educated and deliver the best diagnosis based on an anonymous multidisciplinary medical consultation. The specialists who are well experienced in the endemic areas and have encountered many positive cases of COVID-19 can join the specialist users and help in the diagnosis of suspected cases.

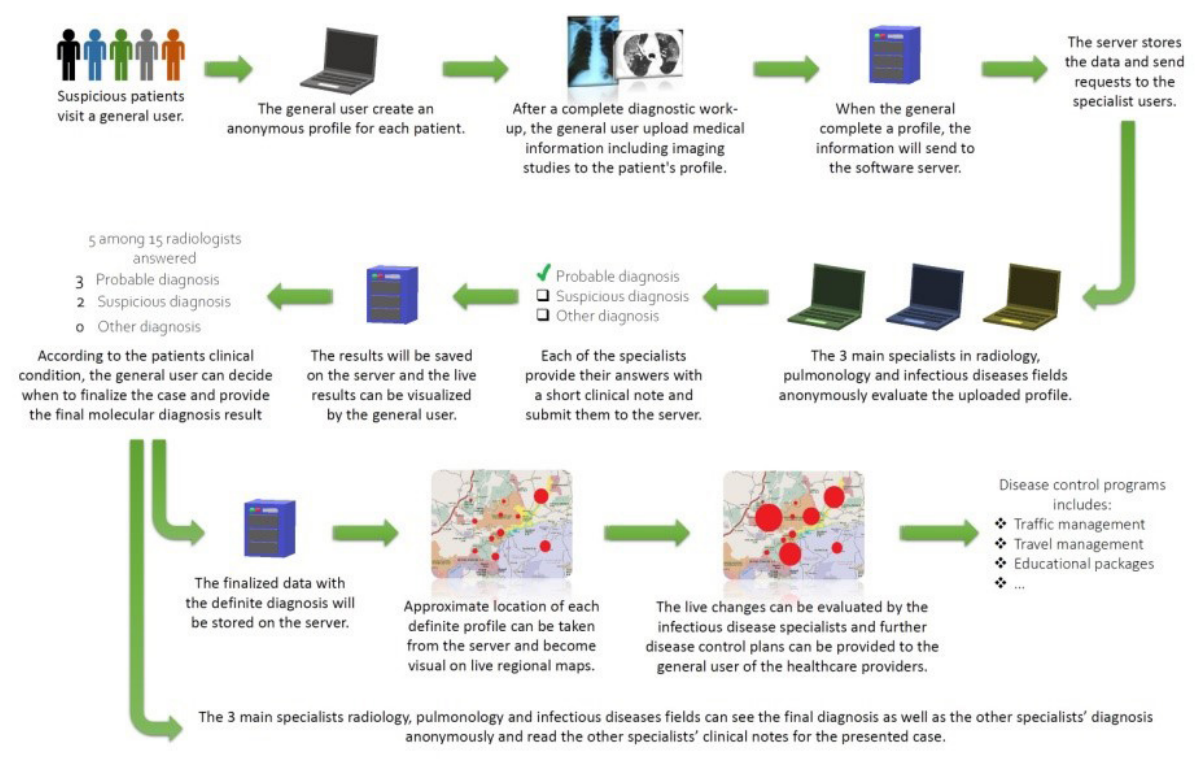

Figure 1. The Flowchart of the Proposed Web-Based Software (Rapid-COVID). The general user is a physician who visits a suspicious case of coronavirus infection. The specialist user is a specialist physician in the field of radiology, pulmonology, or infectious disease. 


\section{Discussion}

Telemedicine and telecare are not widely used during pandemics, including COVID-19; we believe that a software similar to ours can prevent misdiagnosis of patients and also educate the not-well experienced physicians. As molecular diagnostic tests are not available in every clinical setting, recently, diagnostic imaging has become a diagnostic method for determining the infection in both symptomatic and asymptomatic patients. However, similar to many other not well-known diseases, prompt diagnosis of the pulmonary manifestations of this novel diseases in imaging studies require previous experience for at least rolling out the possible differential diagnosis of COVID-19.4 Diagnostic medical imaging in suspicious patients reveals pulmonary involvement in the form of peripheral lesions which include a wide range of differential diagnoses that may be misdiagnosed if interpreted by inexperienced and not well trained medical staff. ${ }^{4}$ We demonstrated that a more accurate diagnosis of such pulmonary lesions could be made through rapid and anonymous online consultations from well-experienced specialists from any country.

In conclusion, new emerging infectious diseases are always considered as a big challenge for health-care providers as there are many unknown diagnostic and therapeutic aspects to cover. The novel coronavirus infection is one such challenge for many societies that still have medical centers that are not well equipped for molecular diagnosis. Such centers only rely on imaging and clinical manifestations. By using such telemedicine platforms, we can ensure that the experience of the specialists will be provided for every suspicious patient in every unequipped center. Moreover, both the counselor and consultant will be educated with any novel clinical manifestation.

\section{Authors' Contribution}

RJE, design the present study and software; MMF, FHHG, ARK and AF; developed the software and validated the study data. MS, ASN, and RM managed the study participants, including the patients and physicians. HRB and MHA supervised the present study. All the authors meet the authorship criteria for enrolling in the present study.

\section{Conflict of Interest Disclosures}

The authors declare no conflict of interest.

\section{Ethical Statement}

The study was approved by the Ethics Committee of Academic Center for Education, Culture, and Research (ACECR)-Khorasan Razavi (IR.ACECR.JDM.REC.1399.001).

\section{Funding}

This research received no specific grant from any funding agency in the public, commercial, or not-for-profit sectors.

\section{References}

1. Zhu H, Wei L, Niu P. The novel coronavirus outbreak in Wuhan, China. Glob Health Res Policy. 2020;5:6. doi:10.1186/ s41256-020-00135-6.

2. The Lancet. COVID-19: protecting health-care workers. Lancet. 2020;395(10228):922. doi: 10.1016/S01406736(20)30644-9.

3. Hollander JE, Carr BG. Virtually Perfect? Telemedicine for Covid-19. N Engl J Med. 2020;382(18):1679-81. doi: 10.1056/ NEJMp2003539.

4. Adhikari SP, Meng S, Wu YJ, Mao YP, Ye RX, Wang QZ, et al. Epidemiology, causes, clinical manifestation and diagnosis, prevention and control of coronavirus disease (COVID-19) during the early outbreak period: a scoping review. Infect Dis Poverty. 2020;9(1):29. doi: 10.1186/s40249-020-00646-x. 